\title{
Characterization of hepatic enzyme activity in older adults with dementia: potential impact on personalizing pharmacotherapy
}

This article was published in the following Dove Press journal:

Clinical Interventions in Aging

14 January 2015

Number of times this article has been viewed

\author{
Noll L Campbell ${ }^{1-4}$ \\ Todd C Skaar ${ }^{5}$ \\ Anthony J Perkins ${ }^{2}$ \\ Sujuan $\mathrm{Gao}^{2,3,6}$ \\ Lang $\mathrm{Li}^{7}$ \\ Babar A Khan $2,3,5$ \\ Malaz A Boustani ${ }^{2,3,8}$ \\ 'College of Pharmacy, Purdue \\ University, West Lafayette, ${ }^{2}$ Indiana \\ University Center for Aging Research, \\ ${ }^{3}$ Regenstrief Institute, ${ }^{4}$ Department \\ of Pharmacy, Eskenazi Health Services, \\ ${ }^{5}$ Division of Clinical Pharmacology, \\ Department of Medicine, ${ }^{6}$ Department \\ of Biostatistics, ${ }^{7}$ Department of \\ Medical and Molecular Genetics, \\ Indiana University School of \\ Medicine, ${ }^{8}$ Center for Innovation \\ and Implementation Science, Indiana \\ University, Indianapolis, IN, USA
}

Objective: To determine the frequency of pharmacogenomic variants and concurrent medications that may alter the efficacy and tolerability of acetylcholinesterase inhibitors (AChEIs).

Materials and methods: A multisite cross-sectional study was carried out across four memory care practices in the greater Indianapolis area. Participants were adults aged 65 years and older with a diagnosis of probable or possible Alzheimer's disease (AD) $(n=105)$. Blood samples and self-reported medication data were collected. Since two of the three AChEIs are metabolized by cytochrome P450 (CYP)-2D6, we determined the frequency of functional genetic variants in the CYP2D6 gene and calculated their predicted CYP2D6-activity scores. Concurrent medication data were collected from self-reported medication surveys, and their predicted effect on the pharmacokinetics of AChEIs was determined based on their known effects on CYP2D6 and CYP3A4/5 enzyme activities.

Results: Among the 105 subjects enrolled, 72\% were female and 36\% were African American. Subjects had a mean age of 79.6 years. The population used a mean of eight medications per day (prescription and nonprescription). The CYP2D6 activity score frequencies were $0(3.8 \%), 0.5(4.8 \%), 1.0(36.2 \%), 1.5-2.0(51.4 \%)$, and $>2.0(3.8 \%)$. Nineteen subjects $(18.1 \%)$ used a medication considered a strong or moderate inhibitor of CYP2D6, and eight subjects (7.6\%) used a medication considered a strong or moderate inhibitor of $C Y P 3 A 4 / 5$. In total, $28.6 \%$ of the study population was predicted to have reduced activity of the CYP2D6 or CYP3A4/5 enzymes due to either genetic variants or concomitant medications.

Conclusion: Both pharmacogenetic variants and concurrent drug therapies that are predicted to alter the pharmacokinetics of AChEIs should be evaluated in older adults with AD. Pharmacogenetic and drug-interaction data may help personalize AD therapy and increase adherence by improving tolerability.

Keywords: dementia, acetylcholinesterase inhibitor, pharmacogenomics

\section{Background}

Acetylcholinesterase inhibitors (AChEIs) are the primary pharmacologic treatment for Alzheimer's disease (AD). These medications do not alter the natural course of the disease, but they are believed to offer minor improvement in cognitive, functional, and behavioral symptoms associated with AD. ${ }^{1,2}$ Efficacy of the AChEIs may be modified by several factors, including stage of dementia, rate of cognitive decline, medication dose, and titration schedule. ${ }^{1,3}$ Adverse effects related to their procholinergic mechanisms often limit the tolerability of these medications, with nearly a third of
Correspondence: Noll L Campbell
Department of Pharmacy Practice, Purdue University College of Pharmacy, Health Information and Translational Sciences Building, 4I 0 West I0th Street - Suite 2000, Indianapolis, IN 46202-30I2, USA

$\mathrm{Tel}+|3| 7274905$ |

Fax +I 3172749305

Email campbenl@iupui.edu 
patients discontinuing treatment due to adverse effects such as nausea, vomiting, and diarrhea. ${ }^{1}$ Because of the marginal benefit and adverse effect profile, as many as $40 \%$ of $\mathrm{AD}$ patients discontinue treatment with AChEIs. ${ }^{4-6}$

Two factors that may contribute to the tolerability of AChEIs in AD patients are drug-drug interactions and pharmacogenomics. Because of the frequent burden of chronic comorbid disease in the older adult population, many AD patients are routinely prescribed at least five medications, and consume additional over-the-counter medications. ${ }^{7,8}$ Many of these coadministered medications cause drug interactions either through altering the activities of the hepatic drugmetabolizing enzymes ${ }^{9}$ or by pharmacodynamic properties suspected to limit the efficacy of AChEIs. ${ }^{10,11}$ Recent evidence highlights the mechanisms of drug interactions resulting from altered pharmacokinetics through hepatic metabolism. ${ }^{12}$ The specific enzymes involved in the metabolism of medications used in $\mathrm{AD}$, specifically donepezil and galantamine, are cytochrome P450 (CYP) 2D6 and 3A4/3A5 (CYP3A4/5) enzymes. ${ }^{13,14}$ The metabolism of donepezil and galantamine may be influenced by either pharmacogenomic variants or comedications, and these variables may be used to identify those at higher risk of intolerable adverse effects.

Pharmacogenomic variants have been associated with altered pharmacokinetics of donepezil and galantamine. ${ }^{15-17}$ Consequently, both comedications, as well as genetic variations in the drug-metabolizing enzymes, may be important factors contributing to the interindividual variability in the efficacy and tolerability of AChEIs. Prior literature has reported conflicting results when determining the relationship between genetic variations and efficacy outcomes with AChEIs in AD $;^{18-21}$ however, to our knowledge, no published data have described the influence of both pharmacogenomic variants or drug-drug interactions on efficacy or tolerability outcomes. ${ }^{15}$

Our objective in this study was to determine the frequency of pharmacogenomic variants and potential drug interactions that may influence the pharmacokinetics of AChEIs, and ultimately efficacy and tolerability, in older adults with AD. Using CYP2D6 enzyme activity to guide dosing strategies has been shown to improve tolerability with several medications, including antidepressants and antipsychotics. ${ }^{22}$ Personalizing pharmacotherapy could be an approach to improve medication adherence by optimizing efficacy and improving tolerability profiles. We focused on the metabolic factors that are expected to alter medication exposure for donepezil and galantamine, since both are metabolized by hepatic CYP2D6 and CYP3A4/5 enzymes. We hypothesized that the presence of a high frequency of pharmacogenomic variants and drug interactions for AChEIs would support the need to pursue focused approaches to personalize pharmacotherapy and optimize efficacy-to-toxicity ratios for these vulnerable populations.

\section{Materials and methods Population and setting}

The subjects included in this study were the first 105 subjects enrolled in a multisite randomized clinical trial (ClinicalTrials. gov identifier: NCT01362686) to compare the tolerability of AChEIs in older adults with a diagnosis of AD. ${ }^{23}$ The study enrolled patients cared for in a memory care practice from one of four health care systems within the Indianapolis Discovery Network for Dementia. ${ }^{24}$ The study was approved by the institutional review boards for each health care system, including Indiana University-Purdue University of Indianapolis, the St Vincent Health System, and the Community Health Network. All enrolled participants provided informed consent through a legally authorized representative.

Eligible participants included those diagnosed with possible or probable $\mathrm{AD}$ for whom the provider was planning to initiate therapy with AChEIs. Inclusion criteria also required agreement from an informal caregiver to complete study-outcome assessments, access to a telephone, and the ability to understand English. Exclusion criteria consisted of current exposure to an AChEI or prior exposure that resulted in an adverse effect requiring drug discontinuation.

\section{Medication-data collection}

Medication data were collected by telephone interview through caregivers of study participants. Caregivers were asked to provide all medications the participant was taking at the time of the interview, including all prescription and over-the-counter medications. Medications were classified as prescription or over the counter by a clinical pharmacist (NLC) according to marketing status, as approved by the US Food and Drug Administration. Medications with CYP enzyme inhibitor or inducer status were identified using the Indiana University Department of Clinical Pharmacology drug-interaction table. ${ }^{9}$ This resource has been previously used to identify clinically relevant drug interactions and metabolic pathways. ${ }^{25}$ Only medications identified as strong or moderate inhibitors were classified as enzyme inhibitors.

Additionally, medications were identified to have either possible or definite anticholinergic properties based on the Anticholinergic Cognitive Burden (ACB) scale. ${ }^{26}$ Medications with anticholinergic properties block the action 
of acetylcholine at muscarinic receptors, opposing the intended therapeutic effect of the AChEIs. Medications with possible anticholinergic effects were defined as those with serum anticholinergic activity or in vitro affinity to muscarinic receptors, but with no known clinically relevant negative cognitive effects (score of 1 on the ACB scale). Drugs with established and clinically relevant cognitive anticholinergic effects were considered definite anticholinergics (score of 2 or 3 on the ACB scale). The ACB scale has been previously used to identify medications correlated with a higher risk of incident cognitive impairment. ${ }^{27,28}$

\section{DNA extraction}

Blood samples for genotyping were collected in $10 \mathrm{~mL}$ ethylenediaminetetraacetic acid Vacutainer tubes at the time of enrollment into the study. Deoxyribonucleic acid (DNA) was extracted from whole blood using the AutoGenFlex Star automated DNA extractor (AutoGen, Holliston, MA, USA) using the AGF FlexiGene and Blood DNA Finishing Kit (AutoGen) in the Indiana University sample-storage facility DNA-extraction service. Because polymorphic variants are infrequent in $C Y P 3 A 4 / 5$, we analyzed the $C Y P 2 D 6$ gene variants and not the $C Y P 3 A 4 / 5$ variants.

\section{CYP2D6-genotyping and -activity scores}

The CYP2D6 genotypes were determined for alleles CYP2D6*1, *2, *3, *4, *5, *6, *10,*17, *29, and *41 using TaqMan $^{\circledR}$ assays. $^{29}$ The genotyping results for each subject were converted to predicted CYP2D6-activity scores as previously described. ${ }^{30}$ Briefly, each subject's activity score is the sum of the activities assigned to each of the subject's two alleles. The wild-type full-activity alleles $(* 1, * 2)$ were given a score of 1 each, the reduced function alleles $(* 10, * 17, * 29, * 41)$ were given a score of 0.5 , and the nonfunctional alleles $(* 3$, $* 4, * 5, * 6$ ) were given a score of 0 . In the case of subjects with multiple copies (more than two), the multiple-copy allele was multiplied by two and then added to the second* allele. Therefore, the predicted CYP2D6-activity scores ranged from 0 to 3. If the subjects were taking a moderate CYP2D6 inhibitor, their genetic score was multiplied by 0.5 , and if they were taking a strong inhibitor, they were given a score of 0 to arrive at the predicted CYP2D6-activity score.

\section{Analysis}

We report descriptive results of the population for the purposes of reporting frequencies of pharmacogenomic variants and drug interactions from concurrent medications that are expected to influence the $\mathrm{AD}$ drug pharmacokinetics in this population of older adults with AD. Continuous variables are presented as means and standard deviations, with categorical variables presented in percentages. Because our analysis was not focused on the number or type of comorbidities, but rather the number of concomitant medications used by study subjects, we report the number of prescription and over-the-counter medications as a surrogate marker of comorbidity. ${ }^{31}$

\section{Results}

The study population included the first 105 subjects enrolled in the clinical trial for whom we had both medication data and pharmacogenomic genotyping results available for analysis. The subjects were mostly female (72\%), were mixed in educational level (28\% with less than high school education), and had a diverse racial background (36\% African Americans). The population used a mean of approximately eight medications per day, including both prescription and over-the-counter medications. Forty-four (41.9\%) of the subjects were taking at least nine concurrent medications. Only $8 \%$ of subjects cared for in memory care practices used a medication with strong anticholinergic properties (Table 1).

Pharmacogenomic analysis revealed four subjects (3.8\%) with a CYP2D6-activity score of 0 , predicting poor metabolizer phenotypes with no CYP2D6 metabolic activity. Forty-three subjects (40.9\%) had activity scores of 0.5 or 1.0, predicting intermediate metabolizer phenotypes. Fifty-four subjects (51.4\%) had activity scores of 1.5 or

Table I Descriptive report of population characteristics and medications

\begin{tabular}{ll}
\hline Demographic variable & Mean (SD) \\
\hline Mean age, years & $79.6(9.1)$ \\
\% Female & $76(72.4)$ \\
\% African American & $38(36.2)$ \\
Education & \\
$\%<12$ years & $29(28.4)$ \\
$\%$ I2 years & $3 I(30.4)$ \\
$\%>12$ years & $42(41.2)$ \\
Medication utilization & \\
Number of medications per subject & $7.55(4.10)$ \\
Number of prescription medications per subject & $5.36(3.07)$ \\
Number of OTC medications per subject & $2.19(2.03)$ \\
Number with 0 medications & 0 \\
Number (\%) with I-4 medications & $26(24.8)$ \\
Number (\%) with 5-8 medications & $35(33.3)$ \\
Number (\%) with 9-I2 medications & $3 I(29.5)$ \\
Number (\%) with $>$ I2 medications & $13(12.4)$ \\
Number (\%) using any ACB drug & $62(59.0)$ \\
Number (\%) using ACB 2 or 3 & $8(7.6)$ \\
\hline
\end{tabular}

Abbreviations: $\mathrm{SD}$, standard deviation; OTC, over-the-counter; $\mathrm{ACB}$, anticholinergic cognitive burden. 
2.0, predicting extensive metabolizers, and the remaining four subjects $(3.8 \%)$ had activity scores greater than 2.0 , predicting ultrarapid metabolizer phenotypes. The specific CYP2D6 allele frequencies and Hardy-Weinberg values are available in Table S1.

Results of the medication review showed that 19 subjects (18.1\%) were using a medication considered a moderate or strong inhibitor of CYP2D6, and eight subjects $(7.6 \%)$ were taking a medication considered a moderate inhibitor of CYP3A4/5. Eleven subjects (10.5\%) were users of a moderate or strong inhibitor of CYP2D6 that reduced activity scores to poor metabolizer status (Table 2). The majority of the CYP2D6 inhibitors used were sertraline and duloxetine, both considered moderate inhibitors of the CYP2D6 enzyme (Tables 2 and S2). The major inhibitor ofCYP3A4/5 used by the study subjects was diltiazem, a moderate inhibitor of CYP3A4/5 (Tables 2 and S2). Additionally, 34.3\% were using at least one CYP2D6 substrate, and 59.0\% were using at least one CYP3A4/5 substrate (Table S2).
Because hepatically metabolized AChEIs utilize both the CYP2D6 and CYP3A4/5 metabolic pathways, we quantified the number of subjects with reduced activity of either CYP2D6 or CYP3A4/5 due to either pharmacogenomic characteristics or concomitant medications acting as moderate or strong enzyme inhibitors. Our results indicate that approximately $29 \%$ of our study population would have reduced activity of either CYP2D6 or CYP3A4/5 activity due to either pharmacogenomic variants or concomitant medications (Table 2). Two of the subjects (1.9\%) were predicted to have reduced activity of both CYP2D6 and CYP3A4/5 (Table 2).

\section{Discussion}

Our study found that more than a quarter of our AD patients $(\sim 29 \%)$ were poor metabolizers of AChEIs, due to pharmacogenomic variants or the presence of drug-drug interactions. These findings may partially explain the high rate of adverse events limiting the chronic use of AChEIs. The population

Table 2 Pharmacogenomic and drug-interaction results

\begin{tabular}{|c|c|c|}
\hline \multirow{2}{*}{$\frac{\text { Variable }}{\text { CYP2D6 activity scores }}$} & \multicolumn{2}{|l|}{ Frequency, n (\%) } \\
\hline & Metabolic status: PG only & Metabolic Status: PG + drug \\
\hline \multicolumn{3}{|l|}{ Predicted poor metabolizers } \\
\hline Number with activity score 0 & $4(3.8)$ & $7(6.6)$ \\
\hline \multicolumn{3}{|l|}{ Predicted intermediate metabolizers } \\
\hline Number with activity score $0.25-0.5$ & $5(4.8)$ & $8(7.6)$ \\
\hline Number with activity score $0.75-1.0^{\mathrm{a}}$ & $38(36.2)$ & $43(4 I .0)$ \\
\hline \multicolumn{3}{|l|}{ Predicted extensive metabolizers } \\
\hline Number with activity score 1.5 or 2.0 & $54(5 \mid .4)$ & $43(4 I .0)$ \\
\hline \multicolumn{3}{|l|}{ Predicted ultra-rapid metabolizers } \\
\hline Number with activity score $>2.0$ & $4(3.8)$ & $4(3.8)$ \\
\hline \multicolumn{3}{|l|}{ Drug-interaction results } \\
\hline Number using CYP2D6 inhibitor & $19(18.1 \%)$ & \\
\hline Bupropion ${ }^{\mathrm{b}}$ & 1 & \\
\hline Duloxetine $^{c}$ & 7 & \\
\hline Paroxetine ${ }^{b}$ & 1 & \\
\hline Sertraline $e^{a}$ & 11 & \\
\hline \multicolumn{3}{|c|}{ (one subject used both bupropion and duloxetine, thus sum of drugs is 20 ) } \\
\hline Number using one or more CYP2D6 substrates & $36(34.3 \%)$ & \\
\hline Number using CYP3A4/5 inhibitor & $8(7.6 \%)$ & \\
\hline Diltiazem ${ }^{d}$ & 7 & \\
\hline Verapamild & I & \\
\hline Number using one or more CYP3A4/5 substrates & $62(59.0 \%)$ & \\
\hline \multicolumn{3}{|l|}{ Combination variables } \\
\hline Number with reduced CYP2D6 activity & $24(22.9 \%)$ & \\
\hline \multicolumn{3}{|l|}{ (activity score of $0-0.5$ or using a CYP2D6 enzyme inhibitor) } \\
\hline Number using a CYP3A4/5 enzyme inhibitor & $8(7.6 \%)$ & \\
\hline Number with reduced activity of either CYP2D6 or CYP3A4/5 & $30(28.6 \%)$ & \\
\hline Number with reduced activity of CYP2D6 and CYP3A4/5 & $2(1.9 \%)$ & \\
\hline
\end{tabular}

Notes: ${ }^{\mathrm{a} F o r}$ some drugs, an activity score of 1.0 is considered an extensive metabolizer; ${ }^{\mathrm{b}}$ potent CYP2D6 inhibitor; ${ }^{\mathrm{c}} \mathrm{moderate}$ CYP2D6 inhibitor; ${ }^{\mathrm{d}} \mathrm{moderate}$ CYP3A inhibitor.

Abbreviations: CYP, cytochrome P450; PG, pharmacogenomic. 
included in this analysis represented a real-world sample of older adults enrolled from four health care systems, including a low-income safety-net health care system. The sample reflects a more normal distribution of educational status and racial diversity than what is commonly enrolled in AD studies conducted within purely academic medical centers and AD research centers. Therefore, the suggestion that both pharmacogenomic characteristics and concomitant medications may contribute to differences in tolerability of AChEIs should apply to the majority of the US population with dementia. In our sample, the number of participants using a medication that reduced hepatic enzyme activity was two times higher than the number of subjects with pharmacogenomic causes for reduced enzyme activity.

Recent work in older adults with AD has shown conflicting results on the association of pharmacogenomic characteristics with clinical efficacy of AChEIs. ${ }^{20,21}$ The sources of variability in previously published literature are derived from different durations of treatment, definitions of efficacy outcomes, and populations with varying degrees of disease severity. The majority of the work associating pharmacogenomic characteristics with clinical outcomes in $\mathrm{AD}$ have focused on the efficacy of AChEIs, not tolerability. ${ }^{14,20,21}$ Whether the activity of CYP2D6 enzymes has an impact on efficacy remains an important outcome to determine; however, we believe tolerability should also be pursued as an important clinical outcome. As stated in the Background section, a clinically significant number of patients prescribed AChEIs discontinue these medications, in part because of poor tolerability. Among those categorized as poor metabolizers, and perhaps even intermediate metabolizers, higher serum concentrations of AChEIs may be more likely to induce an adverse effect than any therapeutic benefit of the medication. Whether clinical efficacy is improved with higher serum concentrations remains controversial, but improving tolerability may result in improved medication adherence and better efficacy outcomes.

Our findings that nearly $30 \%$ of older adults with AD may be poor metabolizers of two AChEIs should be validated with adverse-event data in clinical trials. We were not able to include clinical correlation with tolerability, because the clinical trial is ongoing and adverse events are a primary study outcome. Future studies and analyses from existing data sets should correlate pharmacogenomic and drug interactions with tolerability outcomes.

Our results suggest that if exposure definitions only consider pharmacogenomic characteristics, personalized approaches to the prescribing of AChEIs (specifically donepezil and galantamine) may be improved by genotyping hepatic enzyme activity in approximately one in 12 individuals to find those with reduced activity of the CYP2D6 enzyme (activity score 0 or 0.5 ). However, considering the influence that both concomitant medications and pharmacogenomic characteristics may have on hepatic enzyme activity, nearly one in three older adults may benefit from a personalized approach to medication selection. Interestingly, the predominant influence on enzyme activity seen in our population was with the CYP2D6 enzyme, not the CYP3A4/5 enzyme, which has less influence on the metabolism of donepezil and galantamine. ${ }^{15,16}$

In our sample, $42 \%$ were using at least nine medications, and the risk of drug interactions is known to increase with the number of medications consumed, ${ }^{32}$ which further increases the risk of adverse events in this population. Only $8 \%$ used a medication with strong anticholinergic activity, a similar rate to that reported from other specialty care practices. ${ }^{33}$ Gardette et al found that concomitant use of anticholinergic agents increased the likelihood of discontinuation of AChEIs (hazard ratio 4.26, 95\% CI 1.46-12.45). ${ }^{33}$ Although anticholinergics are known to cause a pharmacodynamic interaction, potentially limiting efficacy, some commonly used anticholinergics are also CYP2D6 enzyme inhibitors (paroxetine, hydroxyzine, diphenhydramine) that may also increase the risk of adverse effects of those AChEIs metabolized through this pathway. ${ }^{9}$

Limitations to our findings include a small sample size and the collection of medication data using a caregiver report. Although our sample reflects more diversity than many prior studies in dementia, a larger sample size may improve generalizability. Among patients with dementia, assistance with medication adherence by a caregiver is recommended; therefore, using the caregiver report of medication utilization is a more appropriate resource for medication utilization than self-report. Although no gold standard exists for the evaluation of medication use, self-reporting and possibly caregiver reporting of medication use may overestimate actual medication-administration behavior.

It is unknown whether exposure to multiple medications that are substrates (not enzyme inhibitors) for the same enzyme would further modify the pharmacokinetics of AChEIs. In our study, 34\% of participants were using a medication metabolized through the CYP2D6 pathway, and $59 \%$ used a medication metabolized through the CYP3A4/5 pathway. Future work should determine if the use of multiple substrates of CYP2D6 and CYP3A4/5 enzymes influences exposure to AChEIs. 


\section{Conclusion}

Our results indicate that both pharmacogenomic variants and concomitant medications may affect the pharmacokinetics and ultimately the efficacy and tolerability of AD medications. It will be important to determine the impact of these combined variables on clinical outcomes of tolerability and efficacy. Such findings should strengthen the rational approaches that can be used to personalize the pharmacologic management of older adults with AD.

\section{Acknowledgments}

This work was supported by grant R01HS019818-01 from the Agency for Healthcare Research and Quality. The content is solely the responsibility of the authors, and does not necessarily represent the official views of the Agency for Healthcare Research and Quality. The sponsor had no role in the study design, evaluation, or manuscript development.

\section{Disclosure}

The authors report no conflicts of interest in this work.

\section{References}

1. Birks J. Cholinesterase inhibitors for Alzheimer's disease. Cochrane Database Syst Rev. 2006:CD005593.

2. Campbell N, Ayub A, Boustani MA, et al. Impact of cholinesterase inhibitors on behavioral and psychological symptoms of Alzheimer's disease: a meta-analysis. Clin Interv Aging. 2008;3:719-728.

3. Schneider LS, Mangialasche F, Andreasen N, et al. Clinical trials and late-stage drug development for Alzheimer's disease: an appraisal from 1984 to 2014. J Intern Med. 2014;275:251-283.

4. Olazaran J, Navarro E, Rojo JM. Persistence of cholinesterase inhibitor treatment in dementia: insights from a naturalistic study. Dement Geriatr Cogn Dis Extra. 2013;3:48-59.

5. Brewer L, Bennett K, McGreevy C, Williams D. A population-based study of dosing and persistence with anti-dementia medications. Eur J Clin Pharmacol. 2013;69:1467-1475.

6. Amuah JE, Hogan DB, Eliasziw M, et al. Persistence with cholinesterase inhibitor therapy in a population-based cohort of patients with Alzheimer's disease. Pharmacoepidemiol Drug Saf. 2010;19:670-679.

7. Schubert CC, Boustani M, Callahan CM, et al. Comorbidity profile of dementia patients in primary care: are they sicker? J Am Geriatr Soc. 2006;54:104-109.

8. Nahin RL, Pecha M, Welmerink DB, et al. Concomitant use of prescription drugs and dietary supplements in ambulatory elderly people. J Am Geriatr Soc. 2009;57:1197-1205.

9. Indiana University. P450 drug interaction table. 2011. Available from: http://medicine.iupui.edu/clinpharm/ddis/main-table. Accessed September 17, 2014.

10. Campbell N, Boustani M, Limbil T, et al. The cognitive impact of anticholinergics: a clinical review. Clin Interv Aging. 2009;4:225-233.

11. Boudreau DM, Yu O, Gray SL, Raebel MA, Johnson J, Larson EB. Concomitant use of cholinesterase inhibitors and anticholinergics: prevalence and outcomes. J Am Geriatr Soc. 2011;59:2069-2076.

12. Hines LE, Murphy JE. Potentially harmful drug-drug interactions in the elderly: a review. Am J Geriatr Pharmacother. 2011;9:364-377.

13. Bachus R, Bickel U, Thomsen T, Roots I, Kewitz H. The O-demethylation of the antidementia drug galanthamine is catalysed by cytochrome P450 2D6. Pharmacogenetics. 1999;9:661-668.
14. Varsaldi F, Miglio G, Scordo MG, et al. Impact of the CYP2D6 polymorphism on steady-state plasma concentrations and clinical outcome of donepezil in Alzheimer's disease patients. Eur J Clin Pharmacol. 2006;62:721-726.

15. Noetzli M, Guidi M, Ebbing K, et al. Relationship of CYP2D6, CYP3A, $\mathrm{POR}$, and $\mathrm{ABCB} 1$ genotypes with galantamine plasma concentrations. Ther Drug Monit. 2013;35:270-275.

16. Eisai. Aricept [prescribing information]. Woodcliff Lake, NJ: Eisai; 2013. Available from: http://www.aricept.com/docs/pdf/aricept_PI.pdf. Accessed September 17, 2014.

17. Janssen Pharmaceuticals. Razadyne [prescribing information]. Available from: http:/www.razadyneer.com/sites/default/files/shared/pi/ razadyne_er.pdf. Accessed September 17, 2014.

18. Klimkowicz-Mrowiec A, Wolkow P, Sado M, et al. Influence of rs1080985 single nucleotide polymorphism of the CYP2D6 gene on response to treatment with donepezil in patients with Alzheimer's disease. Neuropsychiatr Dis Treat. 2013;9:1029-1033.

19. Noetzli M, Eap C. Pharmacodynamic, pharmacokinetic, and pharmacogenetic aspects of drugs used in the treatment of Alzheimer's disease. Clin Pharmacokinet. 2013;52:225-241.

20. Seripa D, Bizzarro A, Pilotto A, et al. Role of cytochrome P4502D6 functional polymorphisms in the efficacy of donepezil in patients with Alzheimer's disease. Pharmacogenet Genomics. 2011;21:225-230.

21. Albani D, Martinelli Boneschi F, Biella G, et al. Replication study to confirm the role of CYP2D6 polymorphism rs1080985 on donepezil efficacy in Alzheimer's disease patients. J Alzheimers Dis. 2012; 30:745-749.

22. Hicks JK, Swen JJ, Thorn CF, et al. Clinical Pharmacogenetics Implementation Consortium guideline for CYP2D6 and CYP2C19 genotypes and dosing of tricyclic antidepressants. Clin Pharmacol Ther. 2013;93:402-408

23. Campbell NL, Dexter P, Perkins AJ, et al. Medication adherence and tolerability of Alzheimer's disease medications: study protocol for a randomized controlled trial. Trials. 2013;14:125.

24. Boustani MA, Frame A, Munger S, et al. Connecting research discovery with care delivery in dementia: the development of the Indianapolis Discovery Network for Dementia. Clin Interv Aging. 2012;7:509-516.

25. Otte JL, Skaar TC, Wu J, et al. Medication use in breast cancer survivors compared to midlife women. Support Care Cancer. 2013;21: $1827-1833$

26. Boustani M, Campbell N, Munger S, Maidment I, Fox C. The impact of anticholinergics on the aging brain: a review and practical application. Aging Health. 2008;4:311-320.

27. Campbell NL, Boustani MA, Lane KA, et al. Use of anticholinergics and the risk of cognitive impairment in an African American population. Neurology. 2010;75:152-159.

28. Cai X, Campbell N, Khan B, Callahan C, Boustani M. Long-term anticholinergic use and the aging brain. Alzheimers Dement. 2013;9: 377-385.

29. Love RR, Desta Z, Flockhart D, et al. CYP2D6 genotypes, endoxifen levels, and disease recurrence in 224 Filipino and Vietnamese women receiving adjuvant tamoxifen for operable breast cancer. Springerplus. 2013;2:52.

30. Gaedigk A, Simon SD, Pearce RE, Bradford LD, Kennedy MJ, Leeder JS. The CYP2D6 activity score: translating genotype information into a qualitative measure of phenotype. Clin Pharmacol Ther. 2008;83:234-242.

31. Perkins AJ, Kroenke K, Unutzer J, et al. Common comorbidity scales were similar in their ability to predict health care costs and mortality. J Clin Epidemiol. 2004;57:1040-1048.

32. Gurwitz JH, Avorn J. The ambiguous relation between aging and adverse drug reactions. Ann Intern Med. 1991;114:956-966.

33. Gardette V, Andrieu S, Lapeyre-Mestre M, et al. Predictive factors of discontinuation and switch of cholinesterase inhibitors in communitydwelling patients with Alzheimer's disease: a 2-year prospective, multicentre, cohort study. CNS Drugs. 2010;24:431-442. 


\section{Supplementary materials}

Table SI Cytochrome P450-2D6 genotype frequencies and Hardy-Weinberg (HW) test

\begin{tabular}{|c|c|c|c|c|c|c|c|c|c|}
\hline & $* I$ & $* 2$ & $* 3$ & $* 4$ & $* 6$ & $* 10$ & $* 17$ & $* 29$ & $* 41$ \\
\hline Major allele & 9 & 38 & 105 & 77 & 104 & 72 & 99 & 95 & 91 \\
\hline Heterozygote & 49 & 38 & 0 & 24 & 1 & 28 & 6 & 10 & 12 \\
\hline Minor allele & 47 & 29 & 0 & 4 & 0 & 5 & 0 & 0 & 2 \\
\hline HW P-value & $\diamond$ & $<0.01$ & I & 0.39 & I & 0.45 & I & I & 0.20 \\
\hline
\end{tabular}

Notes: ${ }^{\circ}$ Since the *I allele is a default allele based on the lack of other alleles, the Hardy-Weinberg test was not calculated, as the minor alleles are actually a variety of all other alleles tested.

Table S2 Medications with influence on hepatic enzymes used by study subjects

\begin{tabular}{|c|c|c|c|}
\hline & Enzyme inhibitor & Enzyme inducer & Enzyme substrate \\
\hline \multirow[t]{12}{*}{ CYP2D6 enzyme } & Bupropion (s) & & Carvedilol \\
\hline & Duloxetine (m) & & Clonidine \\
\hline & Fluoxetine (s) & & Codeine \\
\hline & Paroxetine (s) & & Metoprolol \\
\hline & Sertraline $(m)$ & & Nebivolol \\
\hline & & & Ondansetron \\
\hline & & & Oxycodone \\
\hline & & & Propafenone \\
\hline & & & Propranolol \\
\hline & & & Risperidone \\
\hline & & & Tramadol \\
\hline & & & Venlafaxine \\
\hline \multirow[t]{20}{*}{ CYP3A4/5 enzyme } & Diltiazem (m) & Modafanil & Alprazolam \\
\hline & Fluconazole (m) & & Amlodipine \\
\hline & Verapamil (m) & & Atorvastatin \\
\hline & & & Buspirone \\
\hline & & & Codeine \\
\hline & & & Diltiazem \\
\hline & & & Eplerenone \\
\hline & & & Fentanyl \\
\hline & & & Finasteride \\
\hline & & & Lovastatin \\
\hline & & & Methadone \\
\hline & & & Nifedipine \\
\hline & & & Ondansetron \\
\hline & & & Propranolol \\
\hline & & & Quetiapine \\
\hline & & & Risperidone \\
\hline & & & Salmeterol \\
\hline & & & Simvastatin \\
\hline & & & Trazodone \\
\hline & & & Zolpidem \\
\hline
\end{tabular}

Notes: Does not include study drug as enzyme substrate (acetylcholinesterase inhibitors). List includes only strong/moderate enzyme inhibitors, not weak inhibitors or inducers.

Abbreviations: CYP, cytochrome P450; s, strong; m, moderate.

Clinical Interventions in Aging

\section{Publish your work in this journal}

Clinical Interventions in Aging is an international, peer-reviewed journal focusing on evidence-based reports on the value or lack thereof of treatments intended to prevent or delay the onset of maladaptive correlates of aging in human beings. This journal is indexed on PubMed Central, MedLine,

\section{Dovepress}

CAS, Scopus and the Elsevier Bibliographic databases. The manuscript management system is completely online and includes a very quick and fair peer-review system, which is all easy to use. Visit http://www.dovepress. com/testimonials.php to read real quotes from published authors. 